\title{
Coplanar Waveguide Transmitting a Left-Handed Wave
}

\author{
Jan Macháč ${ }^{1}$, Martin Hudlička ${ }^{1}$, Ján Zehentner ${ }^{1}$, Nikolaos G. Spiliotis ${ }^{2}$, Abbas S. Omar ${ }^{2}$ \\ ${ }^{1}$ Czech Technical University, Technicka 2, 16627 Prague 6, Czech Republic, +420 224352279 \\ ${ }^{2}$ University of Magdeburg, PF 4120, 39016 Magdeburg, Germany, +49 391618499
}

\begin{abstract}
This paper presents a new version of a coplanar waveguide that transmits a left-handed wave. The equivalent circuit of the line unit cell has been derived from the shape of the layout. The values of its elements were determined by fitting the dispersion characteristic of the equivalent circuit to the dispersion characteristic of the first left-handed mode of the line calculated by the CST Microwave Studio. This dispersion characteristic was checked by an experiment. The measured frequency dependences of $S_{11}$ and $S_{21}$ fit well the data predicted by simulation. The line is uniplanar and does not contain any lumped elements or vias.
\end{abstract}

\section{INTRODUCTION}

In recent years left-handed (LH) media or metamaterials that show both negative permittivity and negative permeability have been practically realized [1-3]. In general, they can be designed in a planar form as transmission lines [2] or in a volume form [1,3]. The planar form uses suitable inclusions in a hosting line to achieve the lefthanded properties, while the volume form usually utilizes a system of split ring resonators and thin straight wires periodically placed in the hosting material. The unique properties of these materials may have practical applications, e.g., as antennas, phase shifters, hybrid ring couplers, ideal lenses, single mode waveguides, zeroth order resonators, perfect magnetic layers, frequency selective surfaces, etc. Planar metamaterials can be used in microwave circuits [4]. Inclusions as lumped elements imply more complex fabrication, and for this reason planar structures have mostly received considerable attention $[5,6,7]$.

This paper presents the design of a uniplanar lefthanded coplanar waveguide (LHCPW). The structure does not contain any lumped elements. Its layout is a modification of the line described in [5], but is more compact. The line presented here is applied in microwave circuits, whereas the structure in [5] was aimed for the design of a leaky wave antenna. Our line does not utilize a resonant principle as does the line in [7], therefore it is not narrowband. The presented LHCPW was designed, manufactured and measured. The propagation of a LH wave (or a backward wave) is shown in a frequency band the width of which depends on the line proportions. Higher order LH modes can also propagate along the LHCPW. The scattering parameters of the structure were calculated by the CST Microwave Studio (MwS) and then compared with the measured values. The measured and the calculated parameters fit well. The simple equivalent circuit of the unit cell of the LHCPW has been derived from the shape of the layout. The values of its elements were found by fitting the dispersion characteristic of one cell calculated analytically to the characteristic calculated by the MwS.

\section{LEFT-HANDED CPW}

The layout of one cell of the LHCPW follows the idea of an ideal LH transmission line [2]. A purely left-handed transmission line cannot be fabricated. A practical lefthanded transmission line also contains a series inductance due to the current traveling along the line, and a shunt capacitance resulting from the separation of the metal trace from the ground plane. These elements represent the hosting line. The layout of our unit cell is shown in Fig. 1a. The series capacitor is represented by an interdigital capacitor and the parallel inductor by the input impedance of the shortcircuited CPW stubs connected to the ground metallization. These elements are frequency dependent. By periodically translating the unit cell we get the layout of the LHCPW shown in Fig. 1b. The ROGERS RO4003C substrate with permittivity $3.38,0.813 \mathrm{~mm}$ in thickness and metallization $0.035 \mathrm{~mm}$ in thickness was used.

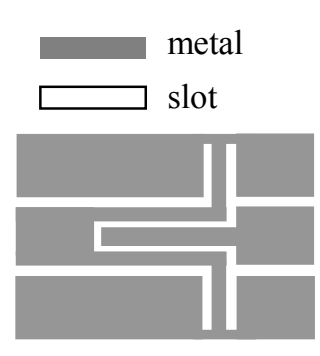

a

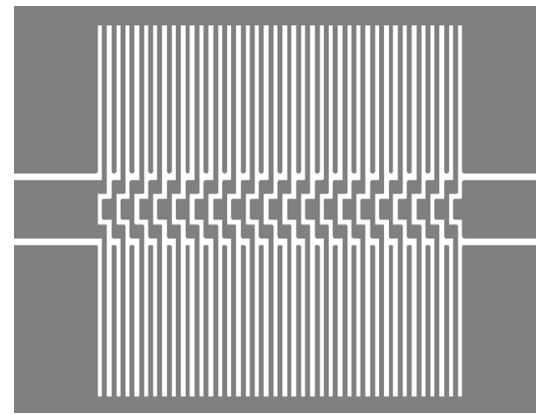

b
Fig. 1 Unit cell (a) and the final layout (b) of the LHCPW.

The dispersion characteristics of modes propagating along the LHCPW drawn in Fig. $1 \mathrm{~b}$ calculated by MwS are plotted in Fig. 2. The calculation was done according to [8]. Firstly, a unit cell of the transmission line was defined and terminated by planes with periodic boundary conditions on both input and output ports with a variable phase shift between these ports. MwS then calculated the resonant frequencies of the cell in dependence on this phase shift. The phase constant of the wave is determined from the known phase shift between the input and output ports, and thus the dependence of 
the resonant frequency on the phase constant (inverse dispersion characteristic) is determined [8]. Each branch of the dispersion characteristic in Fig. 2 defines one pass band of the LHCPW. In the first and third pass band the LH mode propagates, the phase constant decreases and the wavelength increases with frequency. In the second and fourth pass band the right-handed mode propagates, and the phase constant increases with frequency. In this paper, we focus only on the first LH mode.

The frequency dependence of the modulus of the scattering parameter $S_{21}$ is plotted in Fig. 3, and the unwrapped phase of $S_{21}$ is plotted in Fig. 4. The measured values fit well the values computed by MwS. The first left-handed mode propagates from 5.1 to $6.4 \mathrm{GHz}$, as seen in Fig. 2, but the practical transmission band is narrower, from about 5.4 to $6.2 \mathrm{GHz}$. The usable frequency band is about $13 \%$ of the central frequency. The peak of the frequency characteristic, in Fig. 3 at $7.2 \mathrm{GHz}$, corresponds to the propagation of the second, i.e., right-handed, mode. The pass-bands of the third and fourth mode are not shown in Figs. 3 and 4.

Fig. 5 shows the measured dispersion characteristic in the first LH pass band. It agrees well with the calculated characteristics. The phase constant was obtained from the measured wavelength of the standing wave created by the open-circuited termination of the line.

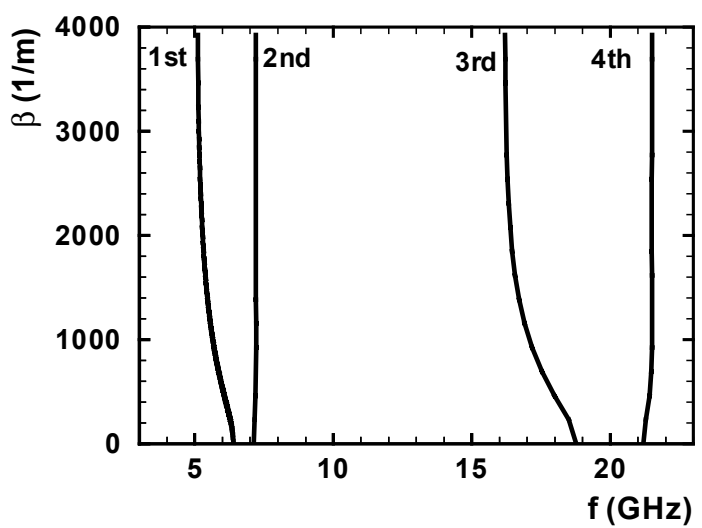

Fig. 2 Dispersion characteristics of the four lowest modes on the LHCPW defined in the text.

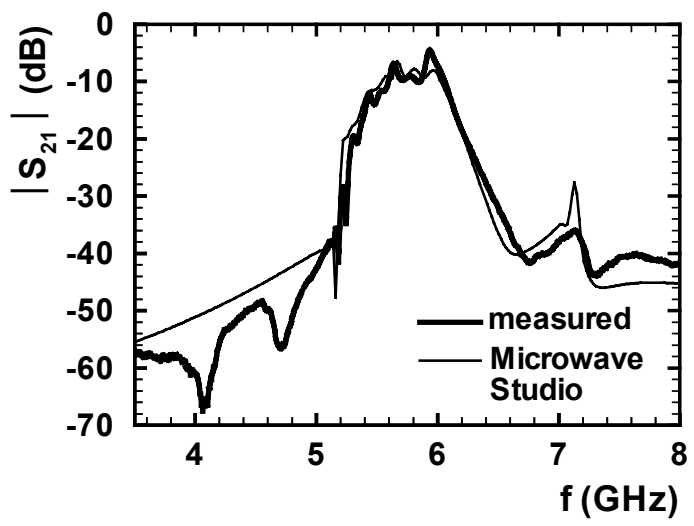

Fig. 3 Measured and calculated modulus of $S_{21}$ of the manufactured LHCPW.

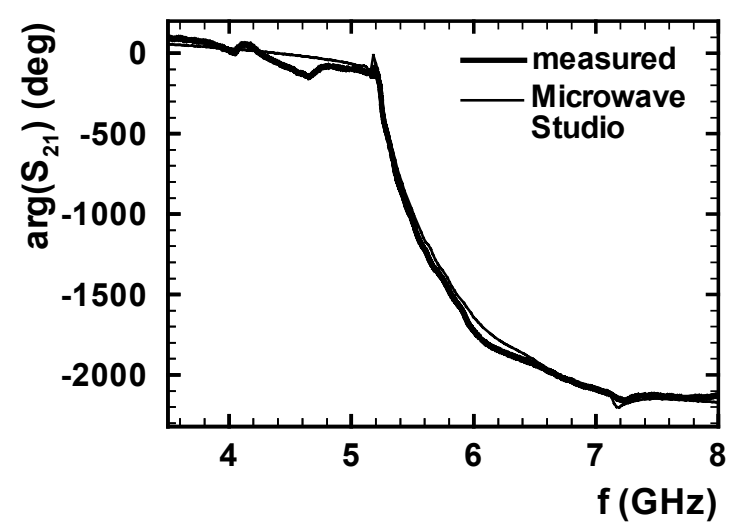

Fig. 4 Measured and calculated phase of $S_{21}$ of the manufactured LHCPW.

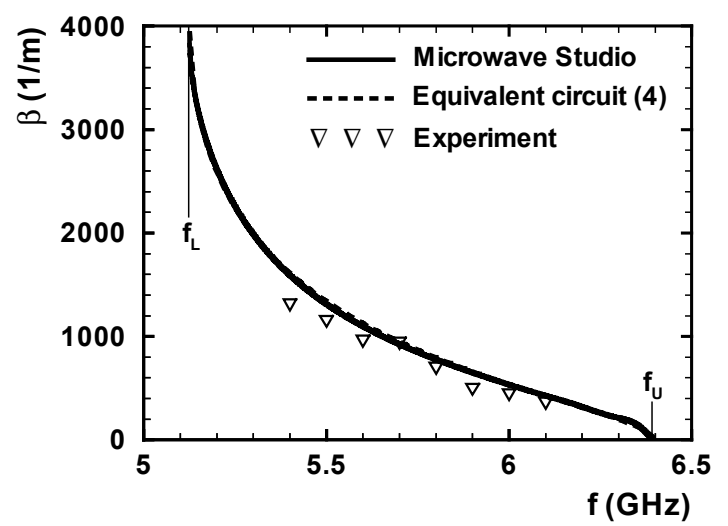

Fig. 5 Comparison of the dispersion characteristics of the fabricated line calculated by the CST Microwave Studio, calculated from the equivalent circuit, Fig. 7, (4) and measured.

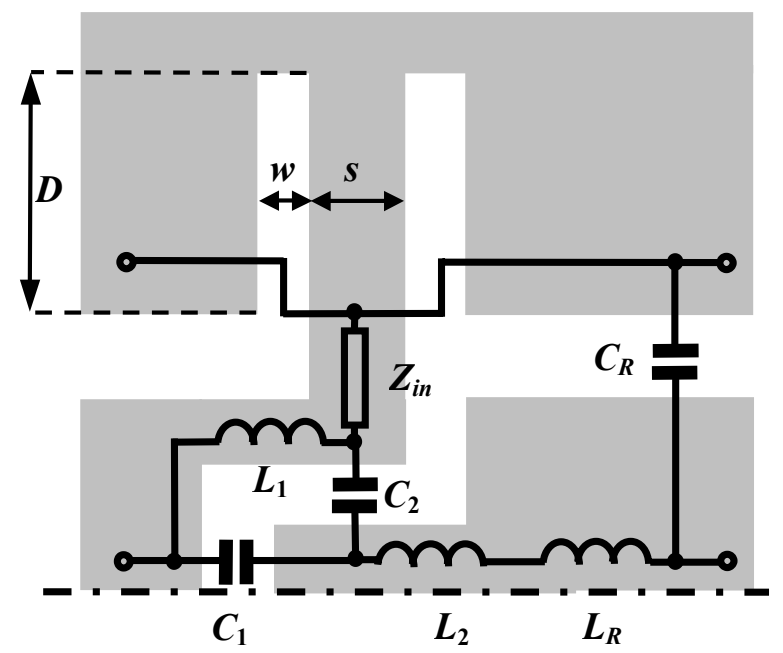

Fig. 6 Simple lumped-element equivalent circuit of one cell of the LHCPW.

\section{EQUIVALENT CIRCUIT OF THE LHCPW}

The equivalent circuit of the LHCPW is shown in Fig. 6, where also one half of the cell layout is depicted. This circuit contains 4 unknowns ( 2 inductors and 2 capacitors) and is able to approximate the dispersion characteristic of the first left-handed mode very accurately. It consists of $L_{R}, C_{R}$ elements representing the hosting $\mathrm{CPW}$ with the characteristic impedance 


$$
Z_{0}=\sqrt{L_{R}^{\prime} / C_{R}^{\prime}}
$$

and the propagation constant

$$
k=\omega \sqrt{L_{R}^{\prime} C_{R}^{\prime}},
$$

where (') denotes the value taken per unit length. The impedance $Z_{\text {in }}$ represents the input impedance of a shortcircuited CPW with finite-extent ground planes of length $D$, which is given by the relation

$$
Z_{\text {in }}=\mathrm{j} Z_{0 e} \tan \left(\beta_{0} D\right)
$$

where $Z_{0 e}$ is the characteristic impedance of the even mode of the CPW with finite-extent ground planes [9], and $\beta_{0}$ is the phase constant of this line. In our case, the stub length is $D=7 \mathrm{~mm}$ and $Z_{0 e}=74.3 \Omega$ and $Z_{\text {in }}$ shows the inductive behaviour.

An ideal left-handed transmission line consists only of series capacitor $C_{1}$ and the parallel inductor formed by the impedance $Z_{i n}$. Parasitic series inductor $L_{2}$ and parallel capacitor $C_{2}$ have been added to the model of the LHCPW layout to make it more realistic. This circuit satisfactorily models the interdigital capacitor and the parallel shortcircuited CPW stubs. The effect of coupling between neighbouring cells is not taken into account. The equivalent circuit is proposed to model only the dispersion characteristic of the first left-handed mode.

The dispersion characteristic is derived using the modified equivalent circuit shown in Fig. 7. The ABCD matrix of the unit cell, Fig. 7, is calculated. Then the dispersion characteristic is obtained by applying Floquet's theorem in the form

$$
\begin{aligned}
& \cos (\beta d)=\frac{1}{2}\left(2+Y_{p}\left(Z_{s 1}+Z_{s 2}\right)\right) \cos \left(k d^{\prime}\right)+ \\
& +\frac{\mathrm{j}}{2 Z_{0}}\left(Z_{s 1}+Z_{s 2}+Y_{p}\left(Z_{0}^{2}+Z_{s 1} Z_{s 2}\right)\right) \sin \left(k d^{\prime}\right),
\end{aligned}
$$

where

$$
\begin{gathered}
Z_{s 1}=\frac{L_{1} / C_{1}}{\mathrm{j} \omega L_{1}+1 /\left(\mathrm{j} \omega C_{1}\right)+1 /\left(\mathrm{j} \omega C_{2}\right)}, \\
Z_{s 2}=\frac{-1 /\left(\omega^{2} C_{1} C_{2}\right)}{\mathrm{j} \omega L_{1}+1 /\left(\mathrm{j} \omega C_{1}\right)+1 /\left(\mathrm{j} \omega C_{2}\right)}+\mathrm{j} \omega L_{2}, \\
Y_{p}=\left(\frac{L_{1} / C_{2}}{\mathrm{j} \omega L_{1}+1 /\left(\mathrm{j} \omega C_{1}\right)+1 /\left(\mathrm{j} \omega C_{2}\right)}+\mathrm{j} Z_{0 e} \tan \left(\beta_{0} D\right)\right)^{-1}
\end{gathered}
$$

The circuit elements $Z_{s l}, Z_{s 2}$ and $Y_{p}$ are obtained by the star-delta transformation of elements $L_{1}, C_{1}, C_{2}$ and by some additional circuit rearrangements.

The values of the lumped elements from Fig. 6 were determined by fitting the dispersion characteristic defined by

(4) to the dispersion characteristic from Fig. 2 calculated by the MwS. The resulting values are $L_{1}=99 \mathrm{nH}$, $C_{1}=0.091 \mathrm{pF}, L_{2}=7.25 \mathrm{nH}$ and $C_{2}=0.34 \mathrm{pF}$.

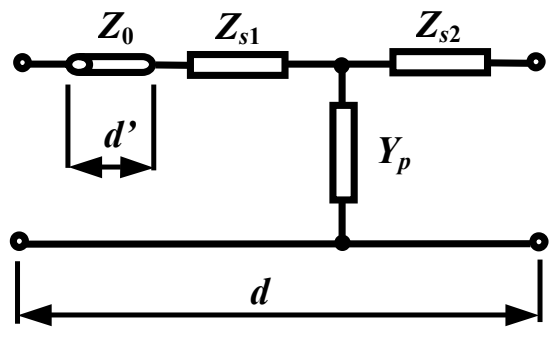

Fig. 7 Modified equivalent circuit of the unit cell of the LHCPW used in the calculation of the dispersion characteristic.

A comparison of the dispersion characteristic calculated by MwS, the characteristic calculated using (4), and the measured characteristic is shown in Fig. 5. The equivalent circuit is quite simple, but it very accurately represents the LHCPW in the frequency band of the first LH mode propagation. At higher frequencies the proportions of the circuit become comparable with the wavelength, and the equivalent circuit gradually losses validity with frequency.
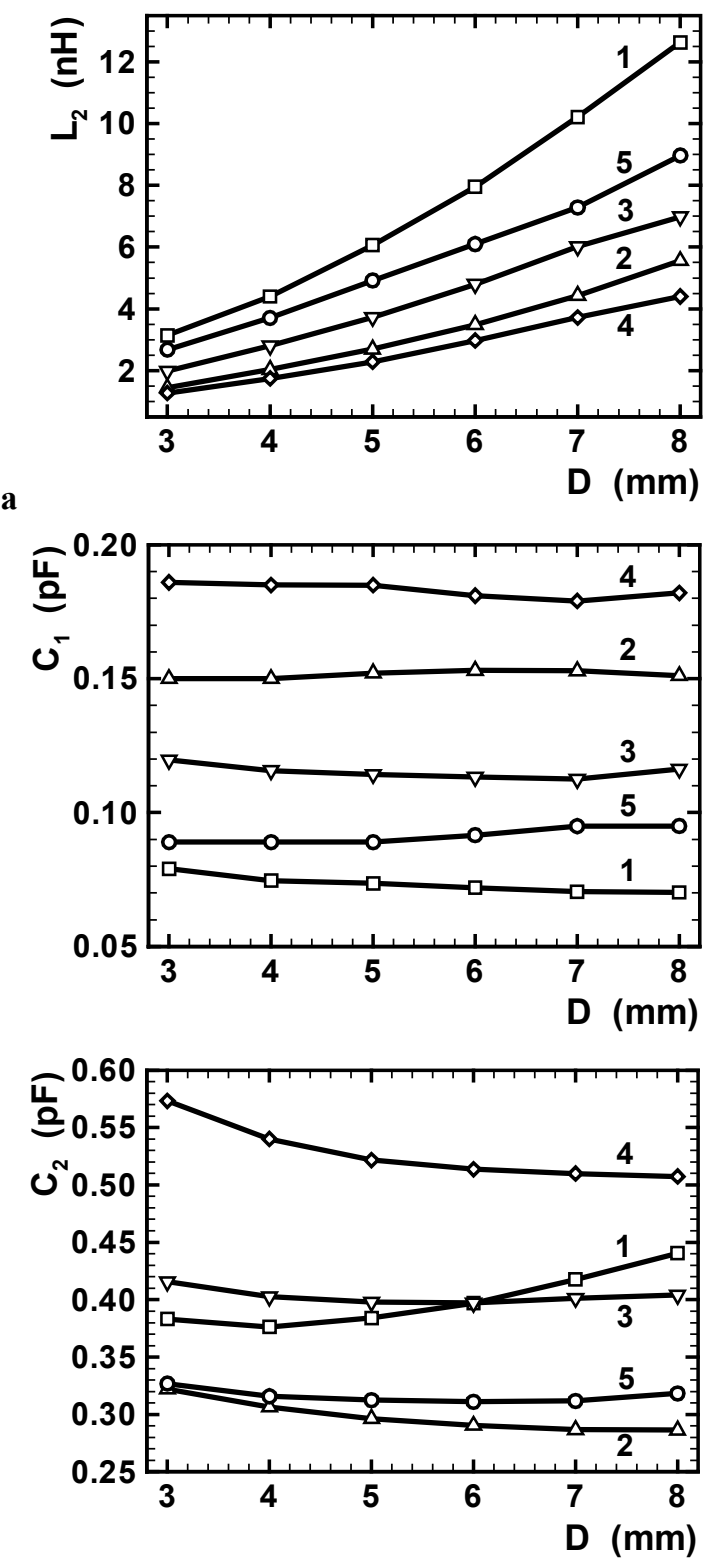

Fig. 8 Values $L_{2}$ - (a), $C_{1}$ - (b), and $C_{2}$ - (c) calculated for different $D, s$, and $w .1-s=0.125 \mathrm{~mm}, w=0.5 \mathrm{~mm}, 2-s=0.125 \mathrm{~mm}$, $w=0.1 \mathrm{~mm}, 3-s=0.25 \mathrm{~mm}, w=0.25 \mathrm{~mm}, 4-s=0.4 \mathrm{~mm}, w=0.1 \mathrm{~mm}$, $5-s=0.125 \mathrm{~mm}, w=0.275 \mathrm{~mm}$. 
To make the equivalent circuit more useful we calculated, with the help of the MwS, the dispersion characteristic of the lowest LH mode propagating on the LHCPW for the number of combinations of the values of the shortcircuited stub length $D$, central conductor width $s$ and slot width $w$, Fig. 6. Each dispersion characteristic was approximated by function (4) and thus $L_{1}, L_{2}, C_{1}$, and $C_{2}$ were obtained. These values are plotted in Fig. 8. Inductance $L_{1}=99 \mathrm{nH}$ is almost constant for all chosen combinations. Changing particular dimensions of the LHCPW layout we change the frequency band of the first LH mode dispersion characteristic. This band is defined in Fig. 5 by lower frequency $f_{L}$ and by upper frequency $f_{U}$. These frequencies are plotted in Fig. 9 as functions of shortcircuited stub length $D$, central conductor width $s$ and slot width $w$, Fig. 6. The frequency band of the LHCPW defined by the transmission characteristic of $S_{21}$ is, however, narrower, Fig. 3.
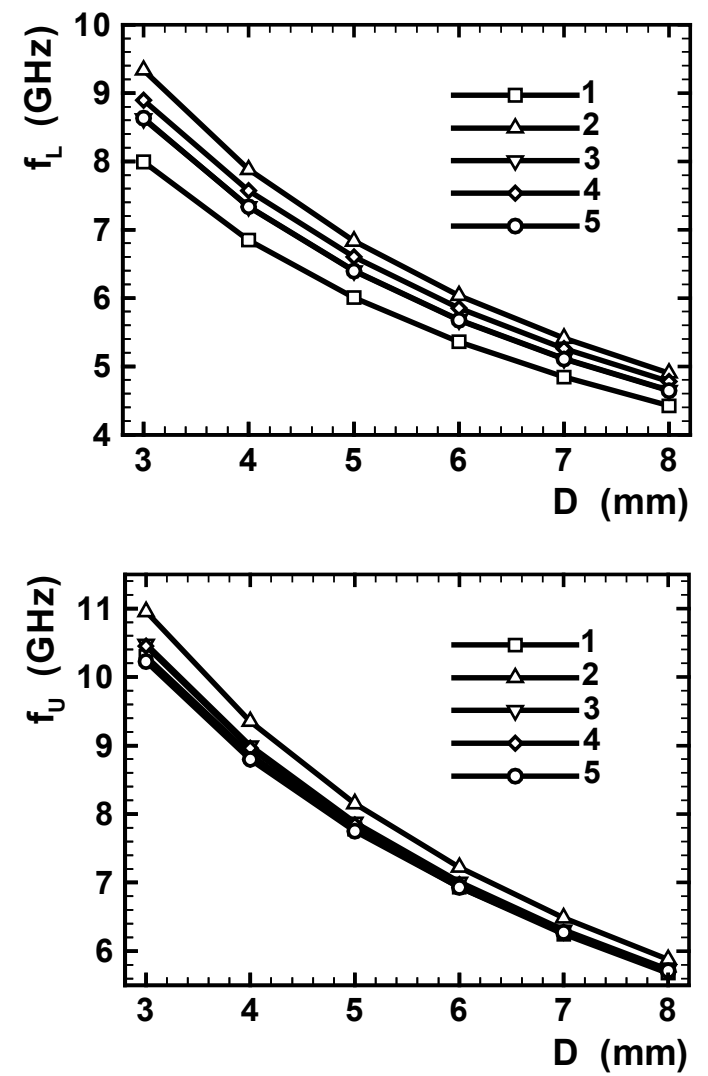

b

Fig. 9 The lower frequency (a) and the upper frequency (b) of the LHCPW dispersion characteristic calculated for different $D$, $s$, and $w .1-s=0.125 \mathrm{~mm}, w=0.5 \mathrm{~mm}, 2-s=0.125 \mathrm{~mm}$, $w=0.1 \mathrm{~mm}, 3-s=0.25 \mathrm{~mm}, w=0.25 \mathrm{~mm}, 4-s=0.4 \mathrm{~mm}$, $w=0.1 \mathrm{~mm}, 5-s=0.125 \mathrm{~mm}, w=0.275 \mathrm{~mm}$.

\section{CONCLUSION}

A new version of the left-handed coplanar waveguide was designed, fabricated and measured. The line has a fully uniplanar structure without lumped elements and vias. The basic left-handed mode propagates in a frequency band about $0.8 \mathrm{GHz}$ in width. The dispersion characteristic calculated by the CST Microwave Studio predicts the pass bands of the left-handed and righthanded modes, which correspond well with the character- istic calculated from the equivalent circuit and the measured transmission characteristic. The simple lumped-element equivalent circuit of the LHCPW valid in the pass band of the lowest LH mode was set up by fitting its dispersion characteristic to the dispersion characteristic of the first lefthanded mode calculated by the CST Microwave Studio. The elements of this circuit were determined for the number of LHCPW structure geometries.

\section{ACKNOWLEDGEMENT}

This research has been sponsored by the Czech Ministry of Education, Youth and Sports in the framework of the project „Research in the Area of Prospective Information and Navigation Technologies" MSM 6840770014, and the experiments were done with support from the Ministry of Culture in Sachsen-Anhalt, Germany.

\section{REFERENCES}

[1] D. R. Smith, W. J. Padilla, D. C. Vier, S. C. Nemat-Nasser, S. Schultz: Composite medium with simultaneously negative permeability and permittivity, Phys. Rev. Lett., Vol. 84, No. 18, May 2000, pp. $4184-4187$.

[2] C. Caloz, T. Itoh: Application of the transmission line theory of left-handed (LH) materials to the realization of a microstrip LH transmission line, IEEE AP-S International Symposium, San Antonio, TX, June 2002, Proc. Vol. 1, pp. 412-415.

[3] R. W. Ziolkowski, C-Y. Cheng: Tailoring double negative metamaterial responses to achieve anomalous propagation effects along microstrip transmission lines, 2003 IEEE MTT-S Digest, Vol. 1, June 2003, Philadelphia, pp. 203-206.

[4] C. Caloz, T. Itoh: Novel microwave devices and structures based on the transmission line approach of metamaterials, 2003 IEEE MTT-S Digest, Vol. 1, June 2003, Philadelphia, USA, pp. 195-198.

[5] A. Grbic, G. V. Eleftheriades: Leaky CPW-based slot antenna arrays for millimeter-wave application, IEEE Trans. Antenna Propag., Vol. AP-50, No. 11, Nov. 2002, pp. 1494-1504.

[6] A. Sanada, K. Murakami, S. Aso, H. Kubo, I. Awai: A via-free microstrip left-handed transmission line, 2004 IEEE MTT-S Digest, Vol. 1, June 2004, Fort Worth, TX, pp. 301-304.

[7] F. Falcone, F. Martín, J. Bonache, R. Marqués, T. Lopetegi, M. Sorola: Left handed coplanar waveguide band pass filters based on bi-layer split ring resonators, Microwave and Wireless Components Letters, Vol. 14, No. 1, January 2004, pp. 1012.

[8] D. R. Smith, D. C. Vier, N. Kroll, S. Schultz: Direct calculation of permeability and permittivity for a left-handed metamaterial, Applied Physics Letters, Vol. 77, No. 14, Oct. 2000, pp. 2246-2248.

[9] G. Ghione, C. U. Naldi: Coplanar Waveguides for MMIC Applications: Effect of Upper Shielding, Conductor Backing, Finite-Extent Ground Planes, and Line-to-Line Coupling, IEEE Trans. Microwave Theory Tech., vol. MTT-35, March 1987, pp. $260-267$. 